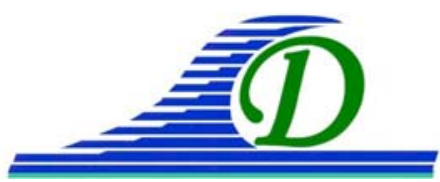

XIII ${ }^{\text {ìmes }}$ Journées Nationales Génie Côtier - Génie Civil Dunkerque, 2-4 juillet 2014

DOI:10.5150/jngcgc.2014.056 @ Editions Paralia CFL disponible en ligne - http://www.paralia.fr - available online

\title{
Modélisation 3D de la dynamique des sédiments fins dans l'estuaire de la Charente (France) : évolution du bouchon vaseux et estimation des flux sédimentaires
}

\author{
Florence TOUBLANC ${ }^{1}$, Isabelle BRENON ${ }^{1}$, Thibault COULOMBIER ${ }^{1}$ \\ 1. Université de La Rochelle, UMR 7266 CNRS - LIENSs, 2 rue Olympe de Gouges, \\ 17000 La Rochelle. florence.toublanc@gmail.com
}

\section{Résumé :}

Les estuaires macrotidaux sont le siège d'une interaction constante entre la marée et le débit fluvial. Les structures sédimentaires qui en résultent varient selon plusieurs échelles temporelles. L'étude menée ici porte plus particulièrement sur la dynamique sédimentaire de l'estuaire de la Charente, situé sur la côte atlantique française. Le bouchon vaseux est notamment reproduit grâce à un modèle numérique hydrosédimentaire 3D. Plusieurs régimes de marée et de débit fluvial sont considérés. La marée reste le processus dominant à l'embouchure, même en cas de crue. Cependant, le débit fluvial a une forte influence sur les caractéristiques du bouchon vaseux (concentration, extension). Les flux sédimentaires cumulés suggèrent qu'en cas de débit moyen, la tendance est à l'import de sédiments dans l'estuaire.

Mots-clés : Estuaire, dynamique sédimentaire, bouchon vaseux, flux sédimentaires, modélisation.

\begin{abstract}
:
In macrotidal estuaries, tides and the river flow are in constant interaction. The consequent sedimentary structures are varying following different temporal scales. This study focuses on the sediment dynamics of the Charente estuary, located on the French Atlantic coast. The turbidity maximum is reproduced using 3D hydrosedimentary modelling. Several tidal and fluvial forcings are considered. Results show that the tide remains the dominant process at the river mouth. However, the river discharge has a strong influence on the turbidity maximum characteristics (concentration, extension). Cumulated sedimentary fluxes suggest that, in the case of an average river discharge, the estuary tends to import sediments.
\end{abstract}

Keywords: Estuary, Sediment dynamics, Turbidity maximum, Sedimentary fluxes, Numerical modelling.

\section{Introduction}

Les estuaires, à l'interface entre océan et continent, sont des systèmes complexes régis par différents processus hydrodynamiques et sédimentaires. L'action conjointe de la 


\section{Thème 2 - Dynamique sédimentaire}

marée et du débit fluvial aboutit notamment à la formation de structures sédimentaires variant selon les cycles tidaux et les variations saisonnières du débit fluvial. Les sédiments fins sont tour à tour déposés et remis en suspension, et une zone d'accumulation, appelée bouchon vaseux, peut se former (ALLEN, 1980). Ces phénomènes ont fait l'objet de nombreuses études dans les grands estuaires du monde (Seine, Gironde, Hudson, Yangtze, Severn, ...). Le déplacement et l'évolution des caractéristiques du bouchon vaseux posent des questions de gestion très importantes (envasement, transport de polluants, qualité de l'eau, ...). Dans ce cadre, l'estuaire de la Charente est une zone d'étude particulièrement intéressante. La dynamique du bouchon vaseux reste à l'heure actuelle peu connue, alors que les activités économiques de la région (ostréiculture, port de commerce de Rochefort) sont fortement dépendantes du bon fonctionnement de l'estuaire. L'objectif de cette étude est donc d'étudier le comportement hydrosédimentaire de l'estuaire (bouchon vaseux et flux sédimentaires) en fonction des variations de régime fluvial et tidal.

\section{Matériel et méthodes}

\subsection{L'estuaire de la Charente}

La côte atlantique française est sous l'influence d'une marée semi-diurne. L'estuaire de la Charente $\left(45^{\circ} 96^{\prime} \mathrm{N}, 1^{\circ} 00^{\prime} \mathrm{W}\right.$, figure 1$)$ est de petite taille, peu profond, et marqué par la présence de vasières intertidales. Le fleuve, long de $365 \mathrm{~km}$, se jette dans la Baie de Marennes-Oléron, dans la partie sud des pertuis charentais. Un barrage est situé $50 \mathrm{~km}$ en amont, à Saint-Savinien.

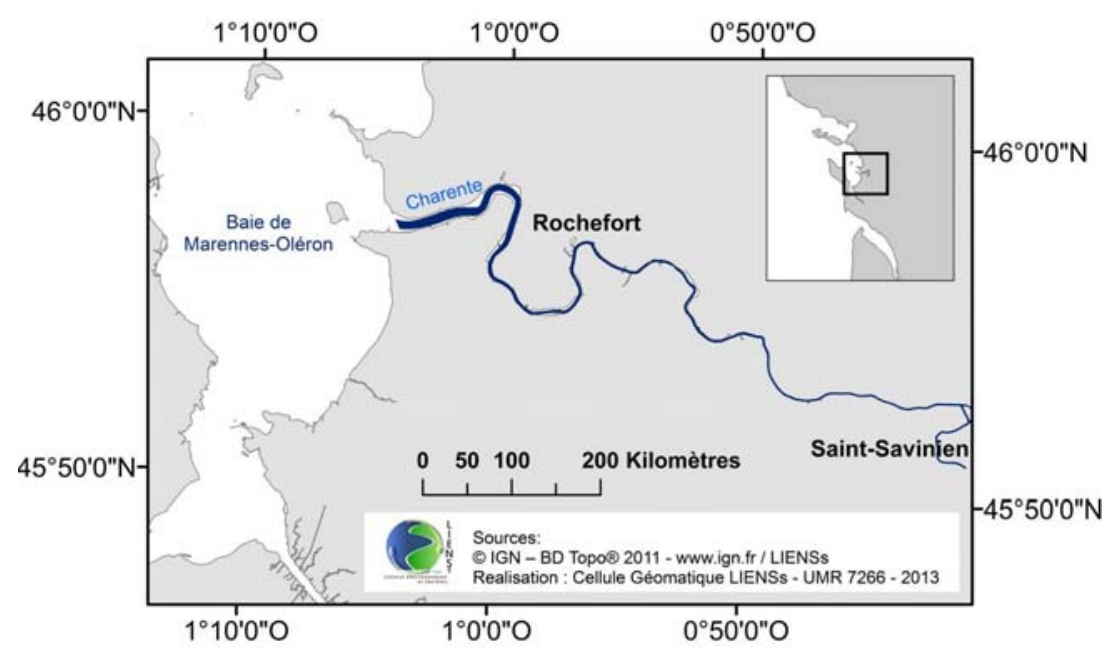

Figure 1. Fleuve Charente, de l'embouchure au barrage de Saint-Savinien. 


\section{XIII ${ }^{\text {èmes }}$ Journées Nationales Génie Côtier - Génie Civil \\ Dunkerque, 2-4 juillet 2014}

La Charente est soumise à un régime de marée macrotidal, avec un marnage moyen et maximal de 4,5 et 6,5 mètres respectivement, à l'embouchure. La marée est caractérisée par des inversions d'asymétrie systématiques suivant le cycle morte-eau/vive-eau (TOUBLANC, 2013). Le débit fluvial moyen est de $70 \mathrm{~m}^{3} / \mathrm{s}$, et peut atteindre plus de $600 \mathrm{~m}^{3} / \mathrm{s}$ dans le cas de fortes crues, ou descendre en dessous de $10 \mathrm{~m}^{3} / \mathrm{s}$ pour des étiages particulièrement sévères.

Les sédiments rencontrés dans l'estuaire et dans la partie orientale de la Baie de Marennes-Oléron sont très majoritairement fins cohésifs (STRADY et al., 2011; TOUBLANC, 2013). La dynamique sédimentaire de l'estuaire est un sujet d'étude particulièrement critique pour la zone, où d'importantes activités économiques sont dépendantes de l'estuaire de la Charente (ostréiculture, navigation commerciale, plaisance, ...).

\subsection{Configuration du modèle}

MARS-3D (Model for Applications at Regional Scales), dont le code est développé par l'Ifremer, est un modèle numérique à surface libre, en différences finies et coordonnées sigma, résolvant les équations classiques de la mécanique des fluides (Navier-Stokes) (LAZURE \& DUMAS, 2008).

Dans cette étude, deux rangs emboîtés ont été utilisés. Le premier rang, d'emprise plus grande, est forcé par une marée issue du modèle CST-France du SHOM (LE ROY \& SIMON, 2003), et permet de fournir les conditions limites pour le rang de résolution plus fine. La bathymétrie et l'emprise de ce rang "Charente" est visible sur la figure 2.

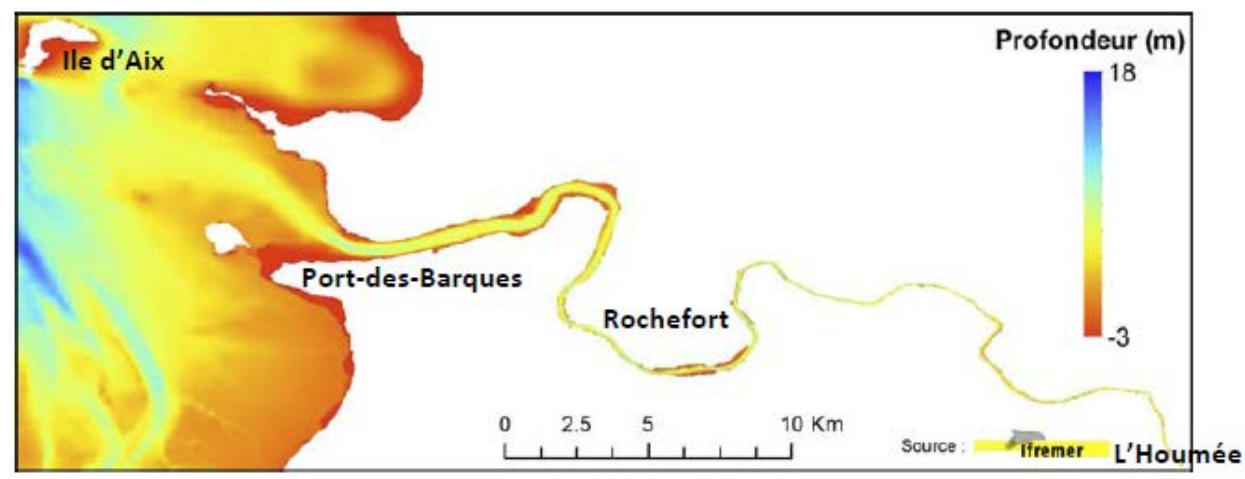

Figure 2. Bathymétrie utilisée pour le rang "Charente".

Les grilles correspondantes à ces rangs, de maillage régulier, ont respectivement des résolutions de 100 mètres $(743 \times 607$ points) et 30 mètres $(1405 \times 766$ points). Les bathymétries utilisées pour ce modèle ont été obtenues à partir de plusieurs sondages réalisés entre 2003 et 2010 (CURTI et al., 2010). Un forçage atmosphérique (ARPEGE, Météo-France) a été utilisé pour les deux rangs. Le débit d'eau douce à la limite amont 


\section{Thème 2 - Dynamique sédimentaire}

est, selon les simulations effectuées, fixe ou variable. Dans ce dernier cas, il est issu des débits journaliers fournis par la Service de Prévision des Crues de la CharenteMaritime. En l'absence de données permettant d'établir une relation débit/concentration en MES, les apports de la Charente sont fixés à $0,05 \mathrm{~g} \mathrm{~L}^{-1}$. Cette valeur a été choisie à partir des mesures effectuées par MODERAN et al. (2012) en amont de l'estuaire.

\subsection{Paramètres sédimentaires}

Les paramètres sédimentaires ont été choisis après une série de tests de calibration et de sensibilité. Au vu de l'uniformité des sédiments rencontrés, le choix a été fait de n'utiliser qu'une classe de sédiments, représentative des sédiments cohésifs vaseux de la Charente. La consolidation n'est actuellement pas prise en compte dans le modèle, et devra faire l'objet de développements futurs. La vitesse de chute des particules varie entre $0,05 \mathrm{~mm} \mathrm{~s}^{-1}$ et $1 \mathrm{~mm} \mathrm{~s}^{-1}$, en fonction de la concentration en sédiments, de la salinité et de la turbulence. Les tensions critiques de dépôt et d'érosion sont fixées respectivement à $1 \mathrm{~N} \mathrm{~m}^{-2}$ et $0,18 \mathrm{~N} \mathrm{~m}^{-2}$.

\subsection{Validation}

Plusieurs données ont été utilisées pour valider le modèle. Les variations de hauteur d'eau ont été validées grâce aux marégraphes de l'île d'Aix et de Rochefort. Les données issues d'un ADCP, placé au niveau de Port-des-Barques ont également été utilisées, afin de valider les hauteurs d'eau et les vitesses de courant. Un ADV, placé au niveau de Rochefort, a permis de compléter ce jeu de données de vitesses. Enfin, des sondes YSI mesurant la salinité et la turbidité ont été mises en place afin d'évaluer la performance du modèle vis-à-vis de ces paramètres.

\section{Résultats}

\subsection{Asymétrie des étales de flot et de jusant}

Les résultats présentés ci-dessous ont été obtenus en simulant des conditions de débit moyen $\left(50 \mathrm{~m}^{3} / \mathrm{s}\right)$ et fixe sur toute la période. Les concentrations en matières en suspension présentées sur les figures 3 et 4 ont été moyennées sur la colonne d'eau, et obtenues pour un cycle de marée de vive-eau. Seules la pleine mer et la basse mer sont présentées, afin d’observer les disparités entre les étales de flot et de jusant. 


\section{XIII ${ }^{\text {èmes }}$ Journées Nationales Génie Côtier - Génie Civil \\ Dunkerque, 2-4 juillet 2014}

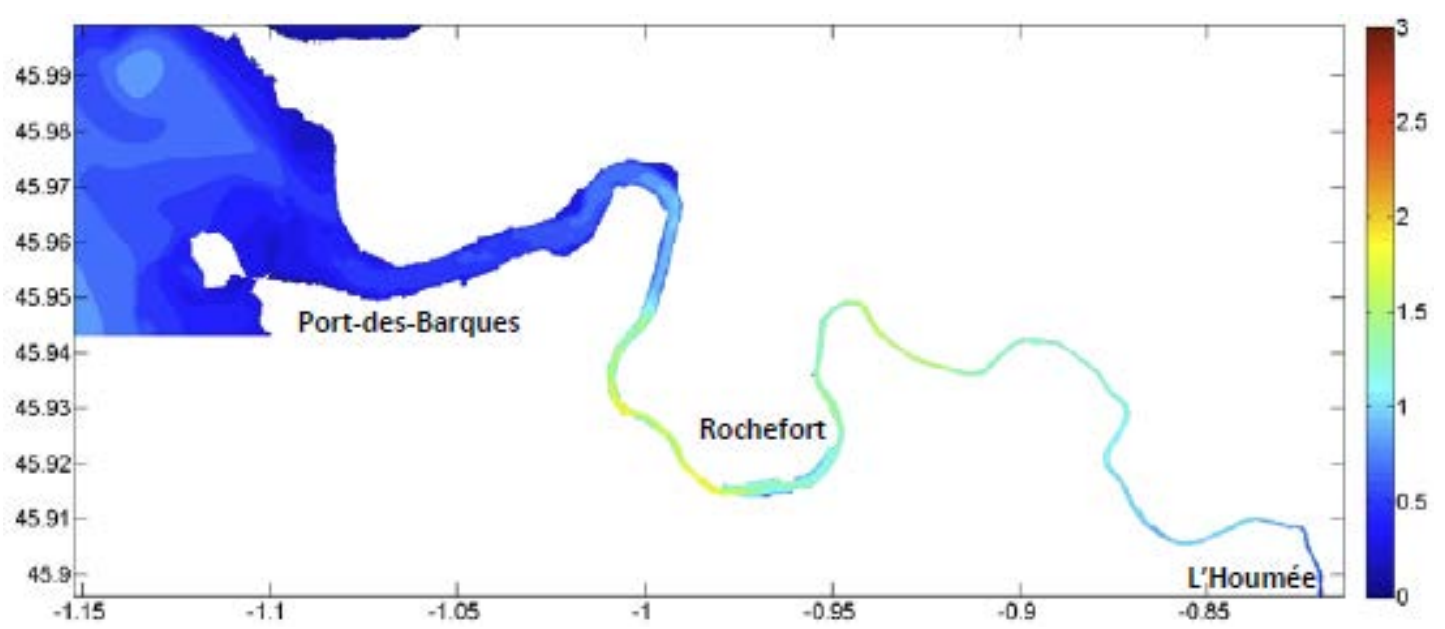

Figure 3. Concentrations en sédiments suspension à marée haute $\left(g L^{-1}\right)$.

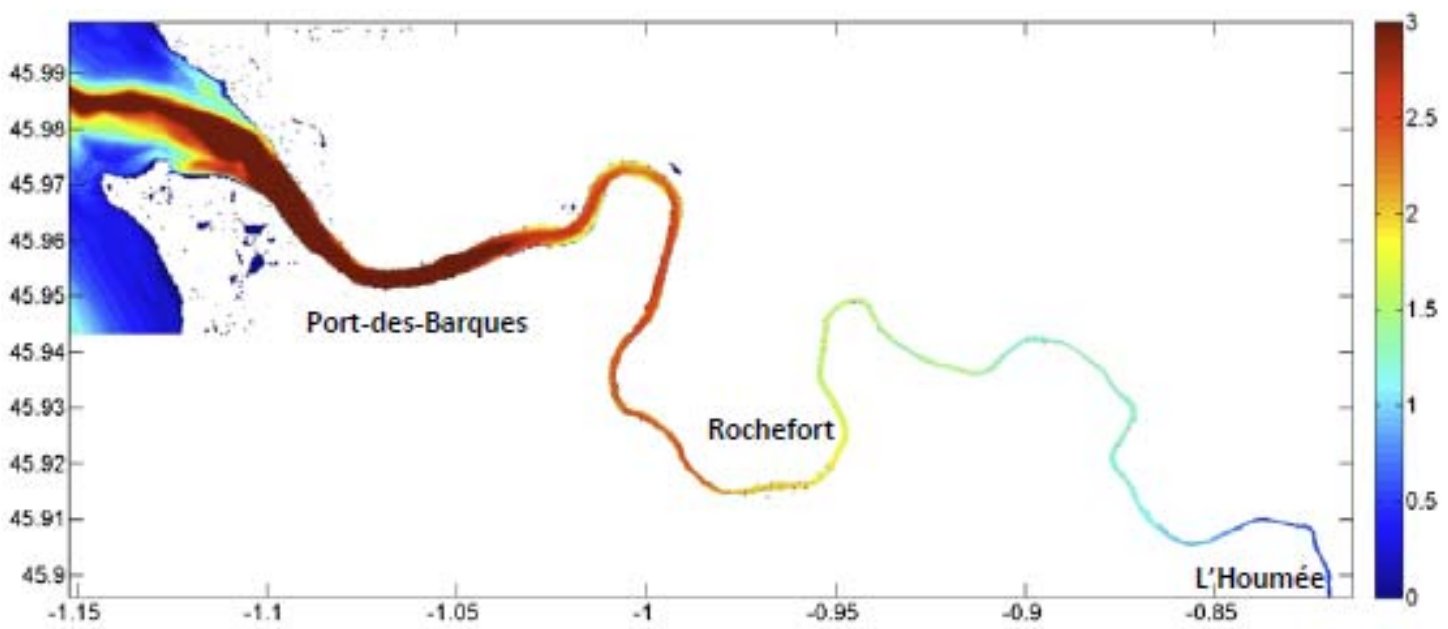

Figure 4. Concentrations en sédiments suspension à marée basse $\left(g L^{-1}\right)$.

En vive-eau, les concentrations en sédiments en suspension les plus faibles sont ainsi obtenues autour de l'étale de pleine mer (figure 3), qui permet aux sédiments de décanter et de se déposer. L'étale de basse mer (figure 4) permet également la sédimentation mais dans une moindre mesure que l'étale de pleine mer. Les concentrations en sédiment sont en effet nettement supérieures à celles obtenues à marée haute (3 fois plus importantes au fond). Cette asymétrie de comportement autour des étales s'explique par la combinaison de fortes vitesses en fin de jusant et en début de flot, favorisant le maintien en suspension des particules. BRENON (1997) et SOTTOLICHIO (1999) ont également noté ce phénomène dans les estuaires de la Seine et de la Gironde. 


\section{Thème 2 - Dynamique sédimentaire}

\subsection{Impact des variations morte-eau/vive-eau}

Les résultats présentés précédemment ont permis de mettre en évidence une forte variabilité sur un cycle flot/jusant de vive-eau. La figure 5 souligne également de fortes variations, mais cette fois à l'échelle d'un cycle morte-eau/vive-eau (débit moyen de $50 \mathrm{~m}^{3} / \mathrm{s}$ ). Les résultats sont présentés de façon à observer les disparités verticales et longitudinales. Les marées hautes et marées basses ont été déterminées à partir des horaires de marée de la station de l'île d'Aix, proche de l'embouchure.

Les maxima représentés par les échelles de couleur sont volontairement différents, afin de pouvoir visualiser les gradients de concentration en vive-eau comme en morte-eau. Les concentrations en sédiments en suspension sont globalement divisées par cinq entre la vive-eau et la morte-eau. Ce résultat est cohérent vis-à-vis des vitesses de courant qui sont plus importantes en vive-eau, appliquant une contrainte plus importante sur le fond et favorisant ainsi les remises en suspension. D’autre part, la turbulence, plus élevée en vive-eau, permet le maintien en suspension de ces particules (DYER, 1997).

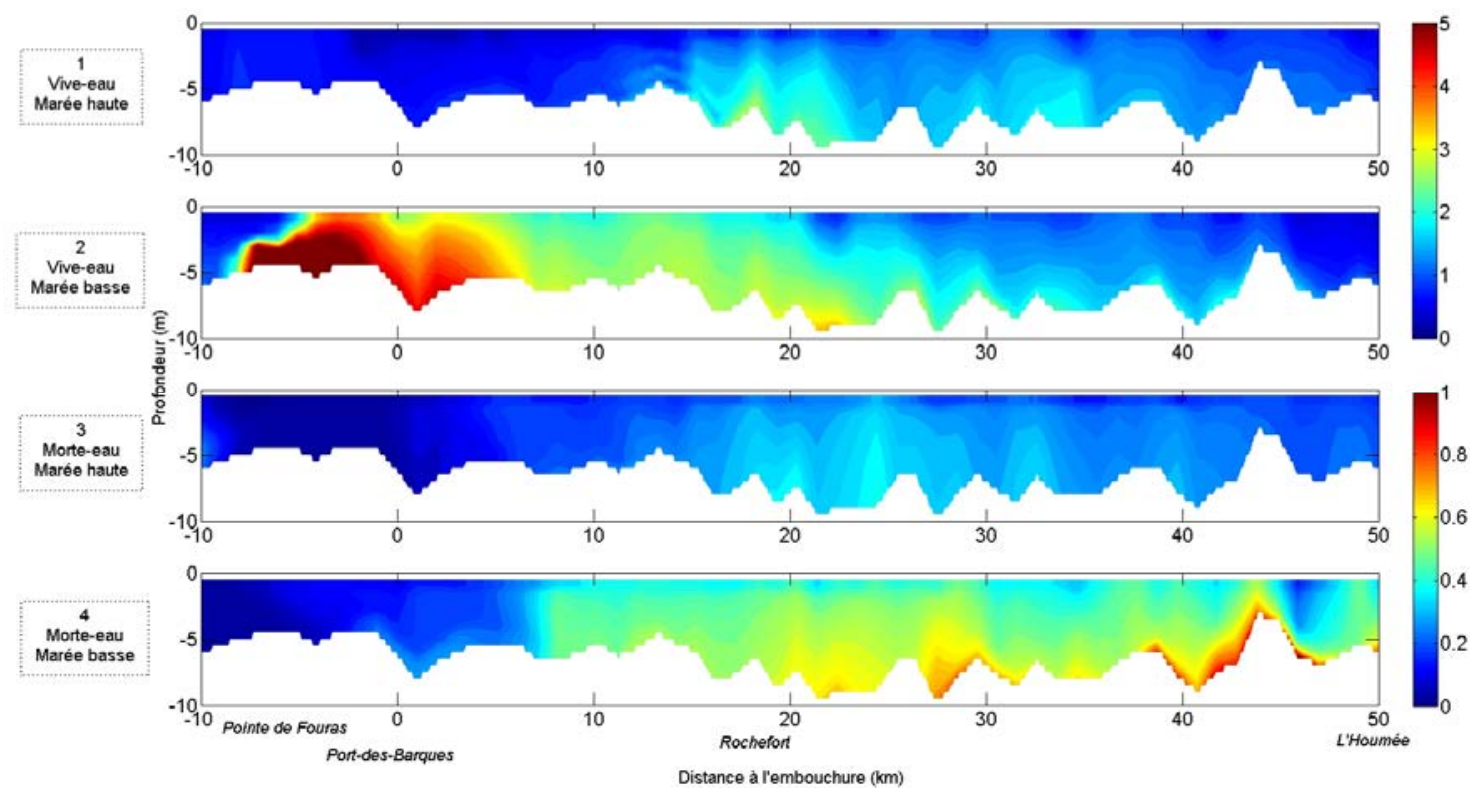

Figure 5. Concentrations en sédiments suspension aux marées basses et marées hautes de vive-eau et morte-eau $\left(g L^{-1}\right)$ - Débit moyen.

A marée basse en vive-eau, les concentrations les plus importantes sont obtenues à l'aval, avec un bouchon vaseux qui semble clairement formé. En morte-eau, les concentrations les plus fortes apparaissent en concordance avec des variations bathymétriques, plus particulièrement en amont. Il est ici difficile de parler de bouchon vaseux, les concentrations étant plus "diffuses", d'aval en amont. 


\section{XIII İmes Journées Nationales Génie Côtier - Génie Civil \\ Dunkerque, 2-4 juillet 2014}

\subsection{Influence du débit}

Les résultats précédents ont permis de mettre en évidence la forte influence de la marée sur les variations de concentrations de sédiments en suspension et les déplacements du bouchon vaseux. L'influence du débit fluvial sur les comportements observés reste à déterminer. La figure 6 illustre des résultats obtenus pour un débit fixé à $400 \mathrm{~m}^{3} / \mathrm{s}$.

En crue $\left(400 \mathrm{~m}^{3} / \mathrm{s}\right)$, la limite amont du bouchon vaseux est plus nette que pour un débit moyen (figure 5). Les concentrations maximales à marée basse en vive-eau sont légèrement supérieures à celles obtenues dans le cas d'un débit moyen, le bouchon vaseux étant plus compact et les sédiments déplacés vers l'aval. En morte-eau, la différence par rapport au régime moyen est nette. Alors qu'il était difficile de parler de bouchon vaseux, on observe ici une masse sédimentaire beaucoup plus formée, avec des concentrations maximales pouvant atteindre $1,7 \mathrm{~g} \mathrm{~L}^{-1}$. Dans les deux cas, le bouchon vaseux ne semble pas être expulsé vers la Baie de Marennes-Oléron, puisqu'il reste contenu au niveau de la pointe de Fouras. La marée reste donc dominante devant le débit fluvial dans la zone de l'embouchure.

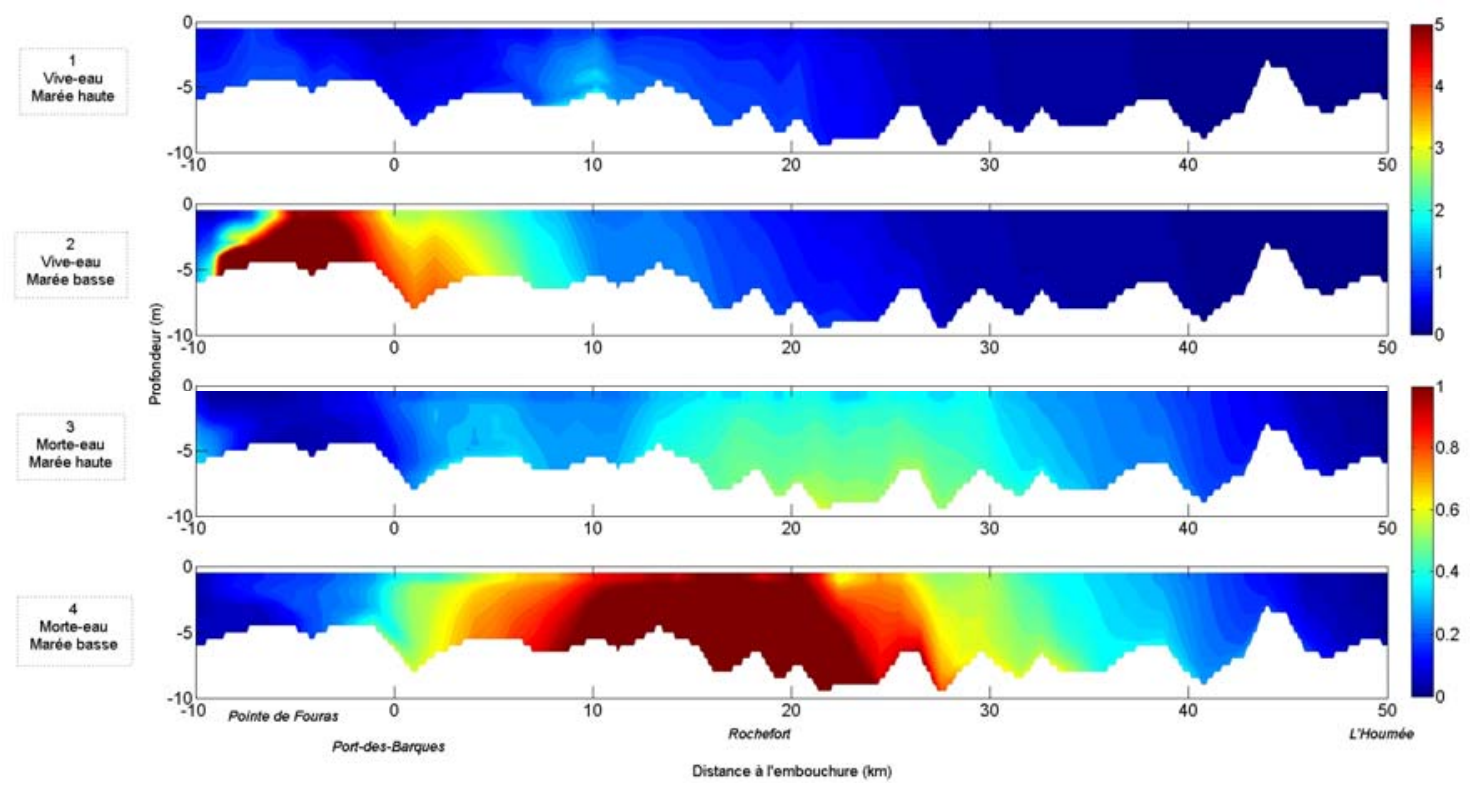

Figure 6. Concentrations en sédiments suspension aux marées basses et marées hautes de vive-eau et morte-eau $\left(g L^{-1}\right)$ - Débit de crue.

Les résultats obtenus dans le cas d'un débit très faible d'étiage $\left(5 \mathrm{~m}^{3} / \mathrm{s}\right)$ montrent que les concentrations en sédiments en suspension sont réduites par rapport au régime moyen. Le forçage fluvial en amont étant quasi inexistant, les remises en suspension associées sont très faibles et ne permettent pas d'alimenter le "bouchon vaseux". Celui-ci est également plus étalé car moins contraint par le débit. 


\section{Thème 2 - Dynamique sédimentaire}

\subsection{Flux sédimentaires}

Les flux sédimentaires cumulés obtenus par modélisation sont présentés en figure 7, dans le cas d'un débit moyen. Ceux-ci sont présentés en kg afin d'évaluer la quantité de sédiments ayant traversé une section du fleuve, depuis le temps 0 . Les deux sections utilisées pour calculer ces flux (figure 7 - haut) ont été choisies afin d'englober le port de Rochefort, infrastructure sensible à l'envasement, et d'observer d'éventuelles disparités de flux entre l'embouchure et la partie amont de l'estuaire.

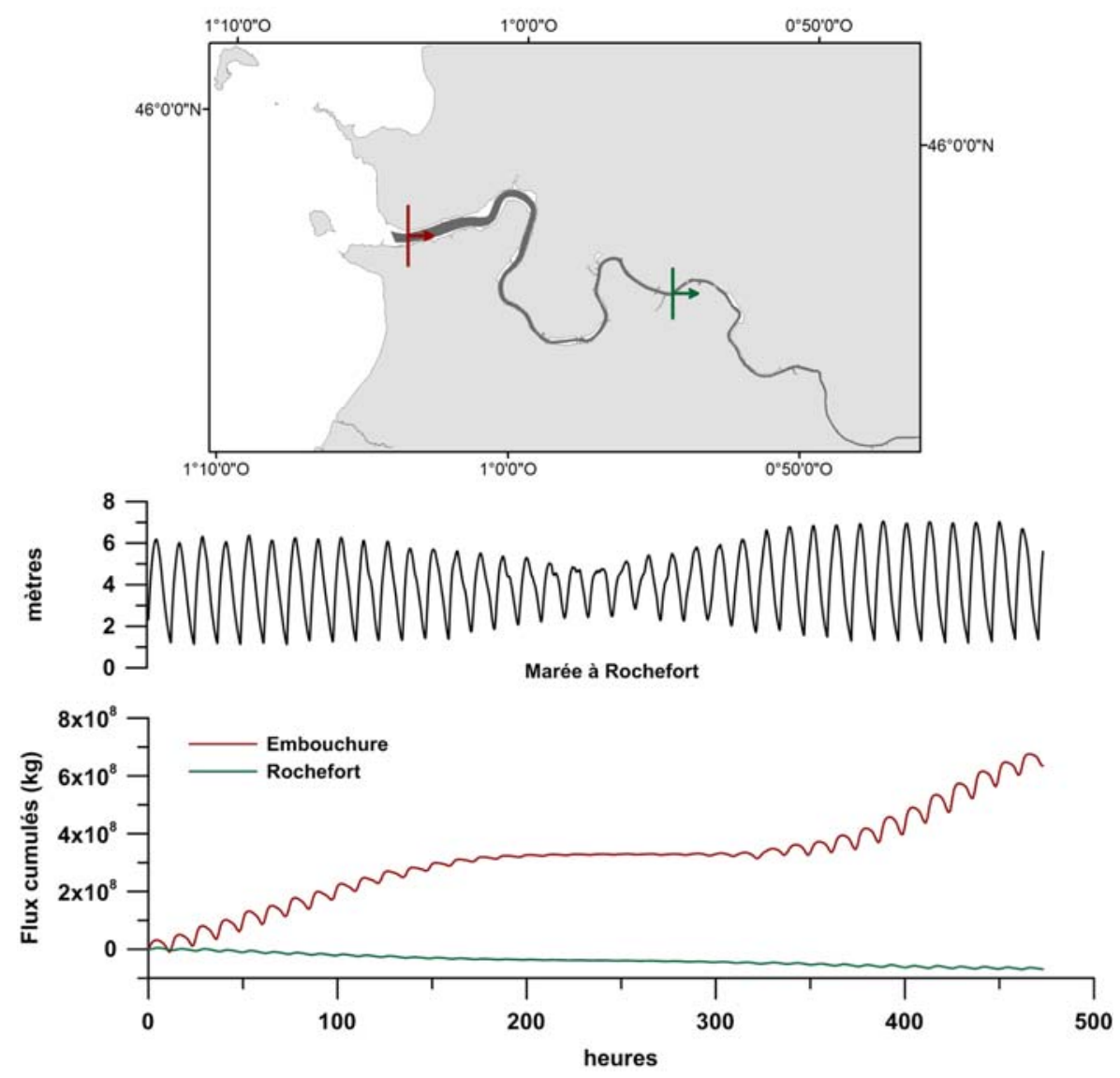

Figure 7. Flux sédimentaires cumulés à l'embouchure et en amont de Rochefort.

Ces résultats montrent un fort import (vers l'amont) sédimentaire à l'embouchure et un faible export (vers l'aval) en amont de Rochefort. La tendance semble donc être à l'accumulation de sédiments dans l'estuaire. Ce résultat est cohérent vis à vis des observations effectuées sur le terrain, qui montrent un envasement global des berges de la Charente (COULOMBIER et al., 2013 ; TOUBLANC, 2013). D'autres études ont mis en évidence cette tendance des estuaires de plaines à l'accumulation de sédiments (GEYER et al., 2001; MEADE, 1969). Plusieurs auteurs ont notamment lié ce comportement à la présence d'une circulation gravitationnelle induisant un transport net 


\section{XIII ${ }^{\text {èmes }}$ Journées Nationales Génie Côtier - Génie Civil \\ Dunkerque, 2-4 juillet 2014}

vers l'amont au fond, là où les concentrations sont en général les plus élevées (GANJU \& SCHOELLHAMER, 2006 ; GEYER et al., 2001).

\section{Conclusions}

La dynamique sédimentaire de l'estuaire de la Charente est fortement dépendante des forçages tidal et fluvial. La modélisation numérique tridimensionnelle a notamment permis de montrer que, dans le cas d'un débit moyen, les concentrations de sédiments en suspension étaient globalement divisées par cinq entre la vive-eau et la morte-eau. En morte-eau, l'existence d'un bouchon vaseux à part entière semble contestable, les concentrations étant relativement diffuses et réparties sur une grande distance.

Cependant, dans les mêmes conditions de marée, mais avec un débit de crue, le bouchon est plus net, avec une masse sédimentaire clairement identifiable. En cas d'étiage, les concentrations sont considérablement réduites. Le débit fluvial a donc une influence non négligeable et il apparaît nécessaire de déterminer ceux-ci avec précision afin de modéliser le comportement hydrosédimentaire de l'estuaire de manière satisfaisante.

Dans tous les cas modélisés, le bouchon vaseux n'est pas expulsé au-delà de $10 \mathrm{~km}$ en aval de Port-des-Barques. L'impact d'une crue exceptionnelle de plus grande importance $\left(600-700 \mathrm{~m}^{3} \mathrm{~s}^{-1}\right)$ reste à déterminer, mais la probabilité d'apparition d'une telle crue sur une période de 15 jours est faible.

\section{Remerciements}

Les auteurs remercient le Conseil Général de la Charente-Maritime et le FEDER pour le financement ayant permis de mener cette étude. Les mesures utilisées pour valider ce modèle ont été acquises avec l'aide précieuse de Nicolas Lachaussée, Philippe Pineau du laboratoire LIENSs de l'Université de La Rochelle et de Florence Cornette, Philippe Geairon, Olivier Le Moine et Stéphane Robert de l'Ifremer-LERPC de La Tremblade.

\section{Références bibliographiques}

ALLEN G.P., SALOMON J.C., BASSOULLET P., DU PENHOAT Y., DE GRANPRE C. (1980). Effects of tides on mixing and suspended sediment transport in macrotidal estuaries. Sedimentary Geology, Vol. 26(1-3), pp 69-90. http://dx.doi.org/10.1016/00370738(80)90006-8

BRENON I. (1997). Modélisation de la dynamique des sédiments fins dans l'estuaire de la Seine. Thèse de doctorat de l'Université de Bretagne Occidentale.

COULOMBIER T., TOUBLANC F., BRENON I. (2013). Seasonal monitoring of sediments dynamics in a highly turbid estuary (Charente Estuary, France): source and sink of the turbidity maximum. Proceedings of the 7th International Conference on Coastal Dynamics, pp 387-396. 
CURTI C., GEAIRON P., LE MOINE O. (2010). Manuel technique : Méthode utilisée pour la mise à jour 2010 de la bathymétrie des pertuis Charentais et du fleuve Charente. Rapport Ifremer.

DYER K. R. (1997). Estuaries: a Physical Introduction. Wiley Ed., London. 140 p.

GANJU N.K., SCHOELLHAMER D.H. (2006). Annual sediment flux estimates in a tidal strait using surrogate measurements. Estuarine, Coastal and Shelf Science, Vol. 69(1-2), pp 165-178. http://dx.doi.org/10.1016/j.ecss.2006.04.008

GEYER W.R., WOODRUFF J.D., TRAYKOVSKI P. (2001). Sediment transport and trapping in the Hudson River Estuary. Estuaries, Vol. 24(5), pp 670-679. http://dx.doi.org/10.2307/1352875

LAZURE P., DUMAS F. (2008). An external-internal mode coupling for a 3D hydrodynamical model for applications at regional scale (MARS). Advances in Water Resources, Vol. 31(2), pp 233-250. http://dx.doi.org/10.1016/j.advwatres.2007.06.010

LE ROY R., SIMON B. (2003). Réalisation et validation d'un modèle de marée en Manche et dans le Golfe de Gascogne. Application à la réalisation d'un nouveau programme de réduction des sondages bathymétriques. Rapport n002/03, EPSHOM.

MEADE R.H. (1969). Landward transport of bottom sediments of the Atlantic Coastal Plain. Journal of Sedimentary Petrology, Vol. 39, pp 222-234.

MODERAN J., DAVID V., BOUVAIS P., RICHARD P., FICHET D. (2012). Organic matter exploitation in a highly turbid environment: Planktonic food web in the Charente estuary, France. Estuarine, Coastal and Shelf Science, Vol. 98, pp 126-137. http://dx.doi.org/10.1016/j.ecss.2011.12.018

SOTTOLICHIO A. (1999). Modélisation de la dynamique des structures turbides (bouchon vaseux et crème de vase) dans l'estuaire de la Gironde. Thèse de doctorat de l’Université Bordeaux 1.

STRADY E., KERVELLA S., BLANC G., ROBERT S., STANISIERE J.-Y., COYNEL A., SCHAFER J. (2011). Spatial and temporal variations in trace metal concentrations in surface sediments of the Marennes Oléron Bay. Relation to hydrodynamic forcing. Continental Shelf Research, Vol. 31(9), pp 997-1007. http://dx.doi.org/10.1016/j.csr.2011.03.006

TOUBLANC F. (2013). Hydrodynamique et dynamique des sédiments fins dans l'estuaire de la Charente. Thèse de doctorat de l’Université de La Rochelle. 